\title{
Acknowledgement of Reviewers for 2020
}

\section{Vassilios A. Tsihrintzis ${ }^{1}$}

Received: 5 January 2021 / Accepted: 5 January 2021/ Published online: 12 February 2021

(C) The Author(s), under exclusive licence to Springer Nature Switzerland AG part of Springer Nature 2021

The Editor-in-Chief and Publisher of Environmental Processes acknowledge the colleagues listed below who provided excellent reviews of papers submitted to the journal during the period January 1, 2020 to December 31, 2020.

$\begin{array}{lll}\text { H. Abdel Ghafar } & \text { S. Al-Saadi } & \text { P. Azeez } \\ \text { M. Abdel Salam } & \text { E.M.E. Alsbou } & \text { N.A.A. Babarinde } \\ \text { R.S. Abdel Tawab } & \text { G.M. AL-Senani } & \text { D. Babu } \\ \text { S. Abdul Rahaman } & \text { M. Al-Shannag } & \text { J. Bai } \\ \text { A.H. Abdullah } & \text { C. Ammer } & \text { R.T. Bailey } \\ \text { S.A. Abed } & \text { J.T. Andersson } & \text { D. Balarak } \\ \text { M.A. Abid } & \text { E.J. Anthony } & \text { M. Baláž } \\ \text { H. Abolghasemi } & \text { C. Anumudu } & \text { L. Baldi } \\ \text { R. Abrutzky } & \text { I. Ara } & \text { F. Ballesteros Jr. } \\ \text { N. Adimalla } & \text { J. Arboleda Zúñiga } & \text { A.M. Banach } \\ \text { H.A. Affum } & \text { J. Archibald } & \text { M. Bao } \\ \text { M.M. Ahammed } & \text { M. Arias-Estevez } & \text { R. Barbato } \\ \text { I. Ahmad } & \text { H. Arslanoglu } & \text { A. Barros } \\ \text { S. Ahmadi } & \text { N. Arunrat } & \text { B. Basak } \\ \text { A. Ahmed } & \text { G. Asadollahfardi } & \text { S.K. Basak } \\ \text { Z. Ai } & \text { S. Ashraf } & \text { F. Battaglia-Brunet } \\ \text { V. Aich } & \text { B. Asl Rousta } & \text { B. Bazargani-Gilani } \\ \text { I. Akoteyon } & \text { C. Asquer } & \text { A. Behnood } \\ \text { H. Akrout } & \text { M.K. Awasthi } & \text { V. Bellos } \\ \text { J.R. Alder } & \text { M.R. Awual } & \text { A. Benchrif } \\ \text { A.A. Alekseeva } & \text { Z. Ayoub } & \text { G.M. Benedetti } \\ \text { I. Ali } & \text { S. Ayoubi } & \text { M. Berenguer } \\ \text { A. Alkaim } & \text { H.M. Azamathulla } & \text { J. Bezzina } \\ & & \end{array}$

Vassilios A. Tsihrintzis

tsihrin@ otenet.gr; tsihrin@survey.ntua.gr

1 Centre for the Assessment of Natural Hazards and Proactive Planning \& Laboratory of Reclamation Works and Water Resources Management, National Technical University of Athens, Zografou, Athens 15780 , Greece 
A. Bhat

H.N. Bhatti

G.S. Bhullar

A.J. Biggs

H. Bilal

D. Bisht

J. Blais

G. Boczkaj

C. Borges

F. Bottino

M. Brestic

M. Brooker

Y. Brouziyne

M.F. Bruno

A. Buffagni

H. Bui

R. Campuzano

N. Cantasano

M. Caporin

R. Carrillo-González

T. Centeno

G. Ceribasi

M. Chatterjee

K. Chau

F. Chemat

C. Chen

J. Chen

L. Chen

Q. Chen

S. Chen

W. Chen

K.Y. Cheng

J.I. Choi

M.F. Chow

A. Chowdhury

Ł. Chrzanowski

J. Coelho

M. Colla L.

J.B. Cousins

E. Couto

J. Cravioto

G. Cruz

J. Cruz-Olivares

B. Cui

K. Cui

M. Dahhou

S.O. Dahunsi
J. Dai

E. Da'na

A. Danandeh Mehr

E. Darakas

S. Das

P. Dave

L.E. Dawidowski

S. De

J. de Brito

S. De Gisi

M.M.G.T. De Silva

A. De Souza

D. Del Buono

J. Deng

R. Devasenathipathy

A.M. Dewan

A. Dey

M. Dharne

L. Di Persio

V. Diamantis

R.J. Diaz

M.S. Díaz-Cruz

E. Dimitriou

P. Ding

S. Ding

P. Drogui

A. Dubnick

S. Dutta

J.N. Edokpayi

N. El Ghachtouli

M. El-Khateeb

I. Elkhrachy

D. Ellison

H.Esbenshade

V. Estelles

M. Evers

A. Fadili

A.A. Faisal

V. Feddern

X. Feng

I. Fernández-Olmo

R.L. Fernando

F. Ferreira

J. Fetzer

M. Field

N. Fiol

P. Fisher
M. Foroughi

B.Y. Fosu-Mensah

R.L. France

S. Frank

M. Fujibayashi

Y. Furuhashi

H. Gao

J.R. García

P. Gassman

C. Gauthier

S.L. Gebre

V. Geraldes

W. Gerbens-Leenes

A. Ghaderi

F. Ghanbari

M.A. Ghorbani

R. Ghosh

E. Gidarakos

G. Gikas

E. Giménez-Forcada

A. Giordani

M. Gmurek

P. Göbel

M. Gocic

D. Gokhale

A.R. Gollakota

W. Gong

M.S. Goni Urriza

M.d.C.A. González-Chávez

F. González-Vidosa

P. Gourbesville

M.K. Goyal

J. Grabić

M. Grattieri

S. Grimaldi

J. Gu

Y. Gu

E.J. Gudiña

R. Guedes

I.C. Guiloski

R. Gumisiriza

I.B.W. Gunam

J. Guo

N. Gupta

V.K. Gupta

V. Gupta

A. Gürses 


\begin{tabular}{|c|c|c|}
\hline L. Gutenberg & K. Jinadasa & S. Kursunoglu \\
\hline L. Gutiérrez-Caminero & H. Jing & S.B. Kusumayudha \\
\hline M. Hachicha & M. Jonoobi & C. Lai \\
\hline Y. Hafez & D.H. Jung & J.C. Lai \\
\hline R. Han & M. Jurado & S.S. Lam \\
\hline M. Hanfi & A.P. Karlapudi & S. Lam \\
\hline A.K.E. Haque & E. Karymbalis & C.W. Lee \\
\hline K. Haribabu & M. Kato & C. Lee \\
\hline E. Haryono & A. Kaur & P. Lee \\
\hline I.A. Hassan & M. Kavianpour & M. Leh \\
\hline M. Hassanshahian & D. Kavitha & S. Lelifajri \\
\hline M. Hatami & G. Kavitha & K.C. Lethesh \\
\hline I. Hatvani & T. Kazi & F. Letto \\
\hline Y. He & $\mathrm{X} . \mathrm{Ke}$ & D. Li \\
\hline S. Heddam & M. Kędra & H. Li \\
\hline M.C. Hespanhol & E. Keramaris & J. Li \\
\hline Y. Но & S.M. Keshk & K. Li \\
\hline L. Höglund-Isaksson & H. Khairy & P. Li \\
\hline L. Hoinaski & K. Khalid & Q. Li \\
\hline N.C. Homem & M.N. Khaliq & Y. Li \\
\hline S.M. Hosseinian & F. Khan & Z. Li \\
\hline V. Hrissanthou & M. Khan & M. Likon \\
\hline C. Hsieh & M.J. Khan & C. Lin \\
\hline W. Huai & M.Y. Khan & J. Lin \\
\hline C. Huang & S. Khandaker & B. Liu \\
\hline L. Huang & T.T. Khidr & D. Liu \\
\hline C. Hwang & A. Khodadad & K. Liu \\
\hline S. Ibarra-Espinosa & T. Khosrawipour & L. Liu \\
\hline H. Ilyas & D. Kidane & Z. Liu \\
\hline M. Iqbal & S. Kim & $\mathrm{X}$. Long \\
\hline M. Iranifam & H. Kiniouar & J. Lopez \\
\hline S. Ismadji & Y. Kiros & R.L. Lorza \\
\hline W. Ismail & V. Kitsikoudis & T. Lotz \\
\hline G. Issabayeva & Z. Kong & A. Loução \\
\hline G. Itskos & Y. Konishi & Y. Lu \\
\hline A. Jaafari & S.B. Kotsiantis & X. Luo \\
\hline S. Jain & N. Kourgialas & A.K.M. Lutfor Rahman \\
\hline F. Jamil & S. Kovács & K. Lyng \\
\hline S. Jamshidi & G. Krishan & J.R. M. \\
\hline B. Janković & R. Krishan & G. Ma \\
\hline M.M. Javed & K.A. Krishnan & X. Ma \\
\hline S. Jena & A.C. Kruger & Y.L. Mack-Vergara \\
\hline J. Jewaratnam & M. Krzywonos & C. Magazzino \\
\hline X. Jia & A. Kumar & A. Mahdavi \\
\hline M. Jian & P.S. Kumar & N. Majed \\
\hline H. Jiang & S. Kumari & Z.A. Majid \\
\hline R. Jiang & A. Kuriqi & S. Makgato \\
\hline
\end{tabular}



M. Malovanyy
G. Mamba
L.D. Manamperuma

S. Maneerat

M. Manzano

Y. Mao

E. Mariño

R. Markovic

A. Marmolejo-Rodríguez

F.M. Márquez-Linares

A.L. Marsh

C. Marsh

E. Marsili

P. Marti

J.L. Martinez

A. Masduqi

M. Masiol

C. Maucieri

I. Mavroidis

Z.A. Mayo

M. McAleer

R. Medeiros

G. Medunic

B. Meikap

W. Meng

R. Merugu

T. Mesbahzadeh

C. Mesquita

J.I. Mikayilov

R.A. Mir

N.D. Miranda Slompo

S. Mirjalili

A. Mishra

S. Mishra

F.M. Mitloehner

M. Mohsenipour

R. Mohtashami

V.P. Molchanov

N. Mondal

P. Monforte

M.L.M. Montenegro

J. Mor

O. Moradi

J. Moreno-Pirajan

E. Morgan Uliana

K. Morisada

Y. Motovilov
G. Moussavi

K. Moutsopoulos

S. Muccifora

R. Mudzielwana

K. Mukesh

I. Mukherjee

J.A. Müller

J. Murillo- Jiménez

S. Myszograj

M.M. Nadzir

W. Nahm

S. Nakao

I. Nalbantis

L. Nandagiri

A. Nanou

M. Narayani

N. Nasirizadeh

R.M. Nasser

M. Naushad

J. Navarro-Laboulais

R.K. Nayak

H. Neidhardt

A. Neisi

Á. Németh

M. Neugebauer

C.C. $\mathrm{Ng}$

F. Nicoletta

J. Noble

N.M. Noor

V. Nourani

S. Ntougias

A. Ogilvie

C.O. Ogunkunle

E.Ohimain

H.A. Okal

V. Oliveira

P. Olupot

D. Orange

O.E. Orisakwe

S. Ouhamdouch

T. Ouyang

W. Ouyang

E. Oyelude

A. Özer

F. Ozyonar

A. Padilla

Z. Padilla-Barrera
C. Paduraru

E. Pagaling

J. Pan

Y. Panagopoulos

P. Pandey

M. Pandya

Y.L. Pang

Y. Pang

H. Pant

N.C. Pant

V. Papaevangelou

J. Parajka

M.J. Park

S. Park

N. Pasukphun

R. Patel

V.P. Patil

W. Patrick

G.V.S.R. Pavan Kumar

G. Pavlikakis

K. Paździor

X. Peng

Y.K. Penke

J. Pérez-Sánchez

R. Peters

J. Plasencia

M. Poletto

S.K. Ponnusamy

H.R. Pourghasemi

O. Pourret

K. Pozo

J.C. Prata

B. Pratap

R. Proshad

T.D.P. Protásio

G. Prpich

M. Przybyłek

A. Rabbani

M. Rafatullah

C. Rafin

M. Rafiqul Islam

E. Rahmadyanti

M.S. Rahman

Z. Rahman

S. Rahman

J.J. Raj

R.S.S. Raja Ehsan Shah 

G. Rakhely
M. Shrestha
M. Tekere
B. Ralph
C. Shu
P. Thabede
K. Ramalingam
S. Siddique
T. Thomas
V. Ramos
S. Silva
P. Thy
M.S. Reddy
J. Silveira
D. Tigkas
M.H. Reis
J. Simal-Gandara
X. Ren
D. Singh
P.V. Timbadiya
A. Rizal
V. Singh
V. Tischer
R. Rodríguez-Soalleiro
R. Singh
A. Tiwari
R. Rostami
E. Sișman
S. Tong
R. Rotz
F. Sitorus
A.J. Toth
S. Roy
S. Sivamani
E. Towler
R. Ruan
P. Sivashanmugam
H.N. Tran
M.J.S. Safari
C. Skoulikaris
V. Tsihrintzis
H. Safavi
I. Skrynetska
B.B. Saha
M. Smiri
J. Saha
S. Sobhanardakani
S. Saha
A.M. Saharia
L. Sobkowiak
E. Sočo
M. Sahoo
M. Solache-Ríos
F.T. Saia
L. Salam
S.A. Solarin
M. Soleimani
C. Salerno
E. A. Soliman
M. Salgot
S. Soni
S. Samanta
M. Soto
V. Sangeetha
S. Squadrone
E. Santero
C. Srinivasakannan
L. Santos-Juanes
P.K. Srivastava
J. T-Thienprasert
N.N.A. Tukimat
V. Tyagi
R. Urban
S. van der Zee
M. van Noordwijk
T.H. Van Hoang
A. VanderZaag
M.C. Varjani
S.J. Varjani
V.S. Varma
P.D.C. Vasconcellos
R. Venkata Rao
A.K. Verma
N. Sarat
T. Sriwiriyarat
J. Verma
J. Vías
R. Saravanathamizhan
C. Stassen
P. Viotti
A. Sargaonkar
A. Stegarescu
C. Viti
M.A.R. Sarker
E. Strogyloudi
D.C. Vodnar
O. Sawai
N. Su
L. Sellaoui
N. Selvaraju
W. Sudjarit
M.M. Vrvic
F.R. Sulaiman
Z. Vryzas
J. Vymazal
T. Setiadi
C. Sun
E.A. Severo
J. Sun
M.T. Walter
S. Shahnawaz
W. Sun
C. Wang
A. Shahzad
B. Sunil
H.Q. Wang
S. Shameer
G. Sylaios
J. Wang
M. Sharif
M. Szyszkowicz
Q. Wang
M. Sharma
M. Tadic
Y. Wang
Z. Wang
D.H. Taffa
D. Wei
N.P. Shetti
R. Talchabhadel
Y. Wei
R. Shetty
S. Tanaka
C. Weng
O. Shishkina
Y. Tanaka
E. Wicklein
P.L. Show
V. Tcherdyntsev
F. Widdel 


$\begin{array}{lll}\text { D. Woldetsadik } & \text { Y. Yang } & \text { J. Zhang } \\ \text { E.D. Woumfo } & \text { Z.M. Yang } & \text { Q. Zhang } \\ \text { P. Wu } & \text { Y. Yao } & \text { W. Zhang } \\ \text { W. Wu } & \text { Z. Yaseen } & \text { Y. Zhang } \\ \text { R. Xiang } & \text { Z.M. Yaseen } & \text { Z. Zhang } \\ \text { Y. Xiao } & \text { F. Yazdian } & \text { H. Zhao } \\ \text { H. Xie } & \text { A.R. Yildiz } & \text { Y. Zhao } \\ \text { K. Xie } & \text { H. Yin } & \text { L. Zheng } \\ \text { S. Xie } & \text { T. Yoshikawa } & \text { Q. Zhou } \\ \text { Z. Xie } & \text { K. Yu } & \text { Y. Zhou } \\ \text { C. Xiong } & \text { W. Yu } & \text { Z. Zhou } \\ \text { G. Xu } & \text { J. Yu } & \text { D. Zhu } \\ \text { Q. Xu } & \text { Z. Yuan } & \text { S. Zhu } \\ \text { T.J. Xue } & \text { Z. Yue } & \text { A. Zorpas } \\ \text { F. Yang } & \text { D.A.M. Zaia } & \text { I. Zotou } \\ \text { H. Yang } & \text { K. Zeinalzadeh } & \text { G. Zou } \\ \text { J. Yang } & \text { B. Zhang } & \end{array}$

Publisher's Note Springer Nature remains neutral with regard to jurisdictional claims in published maps and institutional affiliations. 